\title{
A contextual approach for the development of GIS: Application to maritime navigation
}

\author{
Mathieu Petit, Cyril Ray and Christophe Claramunt \\ Naval Academy Research Institute, 29240, Brest Naval BP 600, France \\ \{petit, ray, claramunt\}@ecole-navale.fr
}

\begin{abstract}
The research presented in this paper introduces the principles of a multi-dimensional contextual approach for adaptive GIS. The framework makes the difference beetween the user, geographical and device contexts. The geographical context is modelled according to the location of the user, the region of interest, the extent of the region covered by the diffusion of the data, and the place where the information is processed. This characterization allows for the study of the different contextual configurations, and their impact on the design of mobile services. The framework is applied to maritime navigation.
\end{abstract}

\section{Introduction}

Technological advances observed over the past few years have favoured the emergence of mobile computing as a novel trend for information diffusion. On the one hand, this opens many opportunities for fullfilling the large range of user needs, but on the other hand current mobile systems often suffer from many limitations such as a lack of connectivity, poor interface design and a dramatic lack of memory and computing power.

Nowadays, recent progress made in energy consumption and computing power, display and memory sizes, and interactive tools have reached a threshold that allows GIS research and development communities to explore novel mobile applications and interfaces. One of the most important technological trends is the progressive integration of different communication techniques that allow mobile GIS and appliances to integrate, process and exchange data using wireless communications. Another trend is the integration of geolocalisation systems within mobile appliances that deliver geographical information for embedded GIS applications [1][7]. These technological advances offer new opportunities for the design and development of mobile GIS.

The research presented in this paper introduces a context-aware mobile GIS that integrates adaptive interaction techniques. We define an adaptive GIS as a generic GIS that can be automatically adapted according to several contexts defined by (1) the properties and location of the geographical data manipulated, (2) the underlying categories that reflect different user profiles and (3) the characteristics of the computing systems, supporting web and wireless techniques. This classification has been inspired by a previous work done by Calvary et al. 
[2]. These contexts cover the components of the diffusion of geographical data in wireless environments. The dimensions identified are of different nature as they involve data, computing processes and interfaces, and categories of users.

These requirements are not new when studied individually, but less considered as a whole. For instance, previous work in the field of adaptive GIS introduces a technology-driven approach for an hardware-based interaction medium [6]. Adaptation of an open GIS layer descriptor to specific user needs and contexts have been also studied in [11]. A context-sensitive model for mobile cartography that emphasizes different levels of data adaptation and presentation have been proposed in [9]. In a previous work, we introduced an architecture and real-time services for the diffusion of maritime geographical information, at different levels from the global monitoring of the maritime traffic of a given area [4], to individual services on request [5].

In order to consider the problem from a global point of view, we introduce a research whose objective is to develop an integrated contextual-based architecture that considers these different factors and interrelationships. The framework is developed and applied to maritime navigation, an emerging field of GIS that combines mobility and distributed services. The remainder of this paper is organised as follows. Section 2 introduces our modelling of a context-aware GIS. Section 3 presents a preliminary application of our framework to maritime navigation. Finally section 4 concludes the paper and draws some perspectives.

\section{A Generic model for adaptive GIS}

The main idea behind an adaptive GIS relies in its capacity to automatically derive its content and interface from a changing environment. This assertion raises the following issue: the contextual dimensions should be clearly identified, and supported by flexible and dynamic algorithms, and adaptive computing processes that support interactions with the users. This constitutes a three-level modelling environment (Fig. 1) whose dimensions can be characterized by the user context (i.e. who), the geographical context (i.e. what) and the appliance context (i.e. how).

The relationships between these dimensions constitute the target of an adaptive process and the subject of our modelling approach. On the software and interaction sides, the system should deliver the geographical data the user may interact with. Geographical data is delivered by a generic communication and integration layer whose role is to aggregate different data flows from either realtime infrastructures, or previously stored geographical information, and to provide an homogeneous source to the adaptive GIS. Another objective of this layer is the internal data storage, that is, monitoring and storing incoming data in order to replay sequences of localized events and scenes that present an interest to the user. As this layer is context independent and constitutes the input of the adaptive GIS, data replays should be adapted to various targets. For instance, a whole geographical scene can be simulated off-line for debriefing or learning 


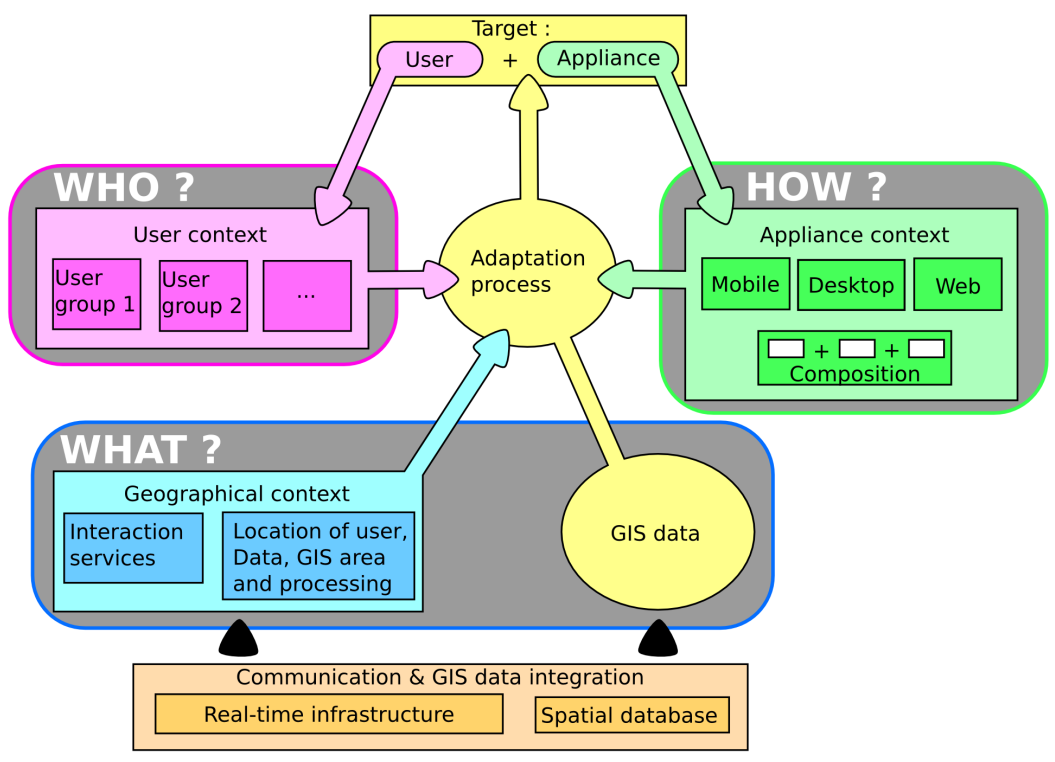

Fig. 1. Context-aware architecture of an adaptive GIS

purposes. A real-time scene can be also enriched with previous scenes to compare current with past situations [3].

This shows that an adaptive GIS constitutes an intermediate layer between data integration and presentation. Within an adaptive GIS, geographical data should be presented to the user with a specific attention to her/his characteristics, and the current context. An adaptation process should integrate elements from different contexts, and apply sorting algorithms to determine the relevant data, and present them in an automatically generated human-computer interface. Taking into account the contextual environment, a mobile GIS should improve the usability and usage of an application. Each contextual class owns its proper means to acquire, characterize and store contextual elements.

The user context reflects the way individual users are sorted into groups of similar behaviours according to the properties of the data usually requested, and the user interface usage. The appliance context characterises the internal specifications (e.g. data transmission speeds and volumes, interface memory), output capabilities (e.g. display size and resolution) and input capabilities (e.g. mouse, touch screen, keyboard). These are the main elements of the appliance context that have to be taken into account by the adaptive process. Appliances are organised into groups of similar capabilities and can be even composed of several devices to support groupwork.

These contextual parameters also constrain the design of the user's interface, that is, the choice and placement of widgets, the user application dialog and the functionalities proposed to the user [10]. 


\subsection{Geographical context}

Geographical data is usually presented to the users by derived views which are available at a given time and space. Each geographical data view is generated by an appropriate service that diffuse its data to different users. As a collection of views, an adaptive GIS is also distributed in space. With respect to the spatial dimension, several locations of significance have been introduced to characterize the geographical context of the execution of a given service [8]:

- $U$ : the location of the user and the interface from where the user obtains GIS-based information. Most of the time, $U$ can be assumed as punctual as the user is likely to be in front of its appliance;

- $D$ : the region of the data is available;

- $P$ : the region where the data is processed;

$-S$ : the region of interest of the GIS project.

Unlike common GIS where $U, P$ and $D$ are static and direectly integrated within the user desktop, in a mobile GIS, locations are distributed and dynamic over space and time. These four orthogonal locations constitute together a multidimensional space whose different configurations can be explored as they are likely to impact the way a given mobile user interacts with geographical data. Our intention is to characterize the range of possible configurations, and to which extent these influence and constrain the services delivered by an adaptive GIS. The range of possible geographical contexts is given by the combination of intersecting and non-intersecting binary relationships between these regions (cf. sample given by Tab. 1).

Table 1. Example of geographical context

When approaching a harbour, a tanker may be guided by an auxiliary vessel, either
from the harbour's authorities or from the tanker itself (Fig. 2(a)). The data from the
region of interest $S$ is sent over a region $D$ covering the surroundings of the harbour.
The tanker processes approaching operations according to the delivered geographical
data. Those processing results are made available into the region $P$ around the tanker.
The user $U$ on the auxiliary vessel may be able to interact with the data through the
tanker processing capabilities. The geographical context and their interactions are
given by: $U \cap D \neq \emptyset, U \cap P \neq \emptyset, D \cap P \neq \emptyset$ and $S \cap D \neq \emptyset$. As $P$ and $U$ are linked
to the mobile vessel and tanker, the geographical context is likely to change. If the
vessel is moving away from the tanker, then at some point $U \cap P=\emptyset$ and the user
will not be able to receive additional data processing results.

In order to represent the possible configurations, a tabular notation is introduced (Fig. 2(b)). Per convention, a black cell represents a non-empty intersection, while a white cell denotes an empty intersection between the location of significance. The combination of these binary spatial relationships generates the 


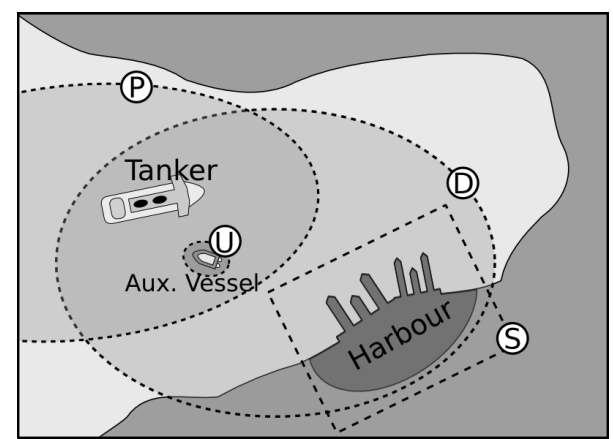

(a) Context-based geographical distribution

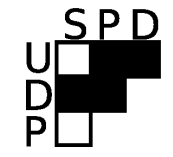

(b) Corresponding geographical and contextual relationships: $U \cap D \neq \emptyset, U \cap P \neq \emptyset, D \cap P \neq \emptyset$ and $S \cap D \neq \emptyset$

Fig. 2. Example of geographical context characterization

complete set of 64 orthogonal contextual configurations presented in Fig. 3. The primitive contextual configurations of a binary relation can be summarized by the following roles:

- $U \cap S \neq \emptyset$ : the user is an actor in the region of interest;

- $D \cap S \neq \emptyset$ : the data is diffused in a part of the region of interest (i.e. local data diffusion);

- $P \cap S \neq \emptyset$ : the process of a service is in a part of the region of interest (i.e. local processing);

- $U \cap D \neq \emptyset$ : the user receives some geographical data as she/he is located in the region of data diffusion (i.e. data reception);

- $P \cap D \neq \emptyset$ : the processing service is available in a part of the region of data diffusion (i.e. service at disposal);

- $P \cap U \neq \emptyset$ : the processing service is available to the user (i.e. service accessed).

The minimum requirements for a service to generate a geographical data view are the simultaneous presence of a service at disposal and accessed by the user. This implies at minimum $D \cap P \neq \emptyset$ and $U \cap P \neq \emptyset ; 16$ combinations out of 64 contextual configurations fullfill this constraint (Fig. 3 - dark grey cells). However, whenever the location of the user intersects other regions (Fig. 3 - light grey cells), these cases are of interest, as an adaptation should occur even when no service is available. For instance, 8 combinations provide no interactions with the user (Fig. 3 - first line) and cannot be considered by an adaptive process. The combination $[c, 4]$ illustrates the desktop use of a non-distributed GIS; all the other combinations denote a certain degree of distribution by the GIS service components. 


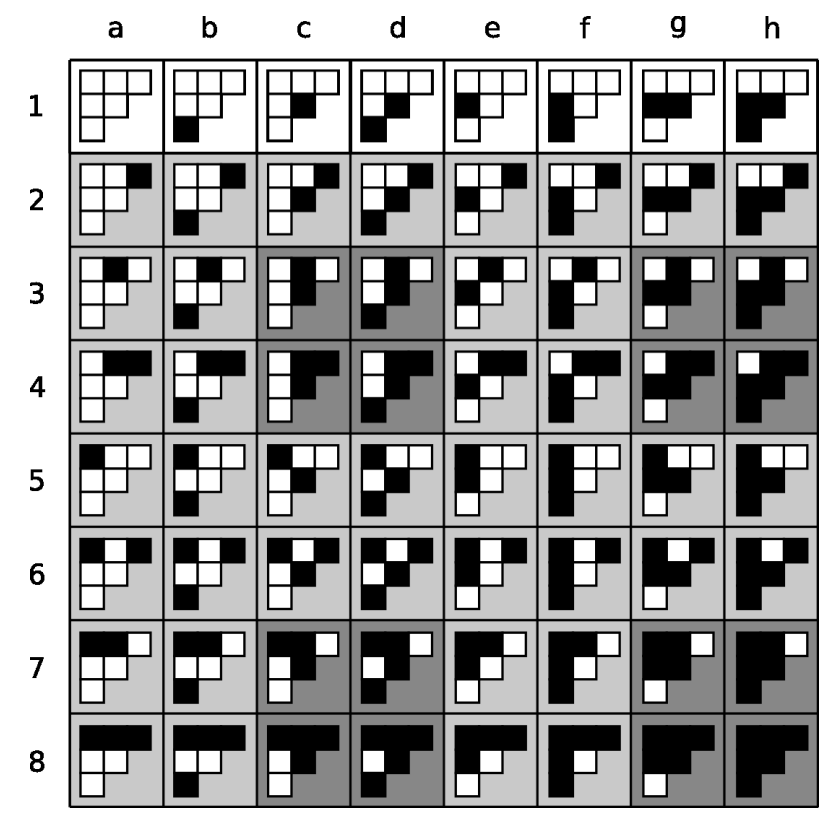

Fig. 3. Possible combinations of the regions of significance into 64 contextual configurations

\section{$2.2 \quad$ Adaptive process}

An adaptive process considers the different contextual parameters, and produces a target-adapted application. The adaptation occurs on the two main components of the adaptive GIS:

- The container, that is, the user interface, by taking into account the user and appliance contexts;

- The content, that is, the geographical data views provided by the services, by considering the characteristics of the geographical context.

The adaptation should be performed at execution time, whenever one of the different contexts change. The user's context is likely to change when the user's behaviour evolves regarding her/his usage of the GIS functionalities and interface. The appliance context should change when the hardware capabilities are modified. The geographical context triggers some adaptive processes when moving from one region to another.

Considering the example proposed in Tab. 1, the user on the auxiliary vessel passes through a series of context cases during the guidance of the tanker to the harbour. Each step corresponds to an adaptation made of a progressive geographical context enrichment. Then a service is made available and is accessed when the data diffusion and processing regions intersect the user location. Fig. 
4 illustrates a possible sequence of contextual configurations changes. Each role within a configuration involves a specific adaptation of the view provided by the service. When the value of another binary spatial relationship is modified, the roles are updated before re-calculation of the view.

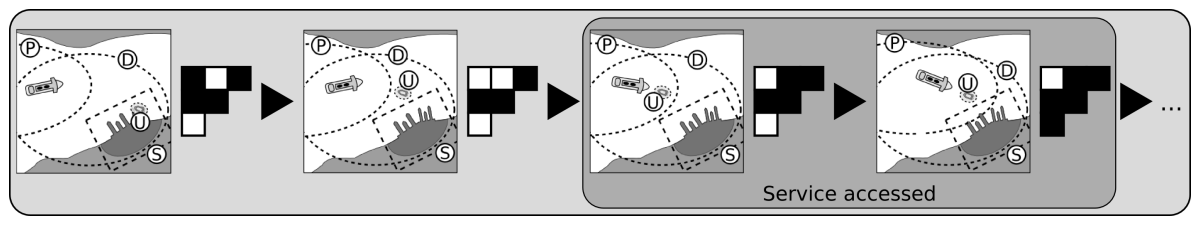

Fig. 4. Example of geographical context evolution : the auxiliary vessel leaves the harbour towards the tanker and drives it back to the harbour.

\section{Maritime navigation: case study}

Our experimental framework has been tested in the context of an international sailing race. This event has a large audience, and requires appropriate solutions to diffuse real-time information, from the coastal maritime area to the users located in the ground. This generates different needs in term of geographical information usage and appliance. The experimental prototype is composed of two parts: a wireless network and an experimental adaptive GIS (Fig. 5).

Ships locations during the race are acquired through a real-time infrastructure. The implementation of the geographical context into different views and an appliance context divided into several classes of devices are considered by the adaptive process. The user context is modelled by a generic user group.

\subsection{Communication and data integration}

An important aspect of the adaptation process is its ability to integrate real-time geolocalisation information that delivers GIS data and influences the geographical context and related services. A localisation system has been developed and allows for real-time reception of ship's positions and a continuous video stream of the race (Fig. 6). locations are provided by an embedded system available on ships. This system includes a GPS, a configurable modem, a VHF transmitter and fulfills several constraints: light weight (less than a $\mathrm{kg}$ ), long range ( 5 to $10 \mathrm{~km}$ ), high autonomy ( 8 to 10 hours). This module collects and diffuses the real-time locations of the ships to the ground station (Fig. 6- $(d)$ ). The transmission to the ground station is a VHF communication (Fig. 6- $(a)$ ) based on APRS frames (Automatic Position Reporting System). The ground station is composed by a VHF receiver and a VHF-to-WiFi bridge that broadcasts real-time data to a given area (Fig. 6- $\left(c_{2}\right)$ ). Mobile end-users (Fig. 6- $(f)$ ) located in this broadcast area can access ships' data, whatever the form of their appliance. 


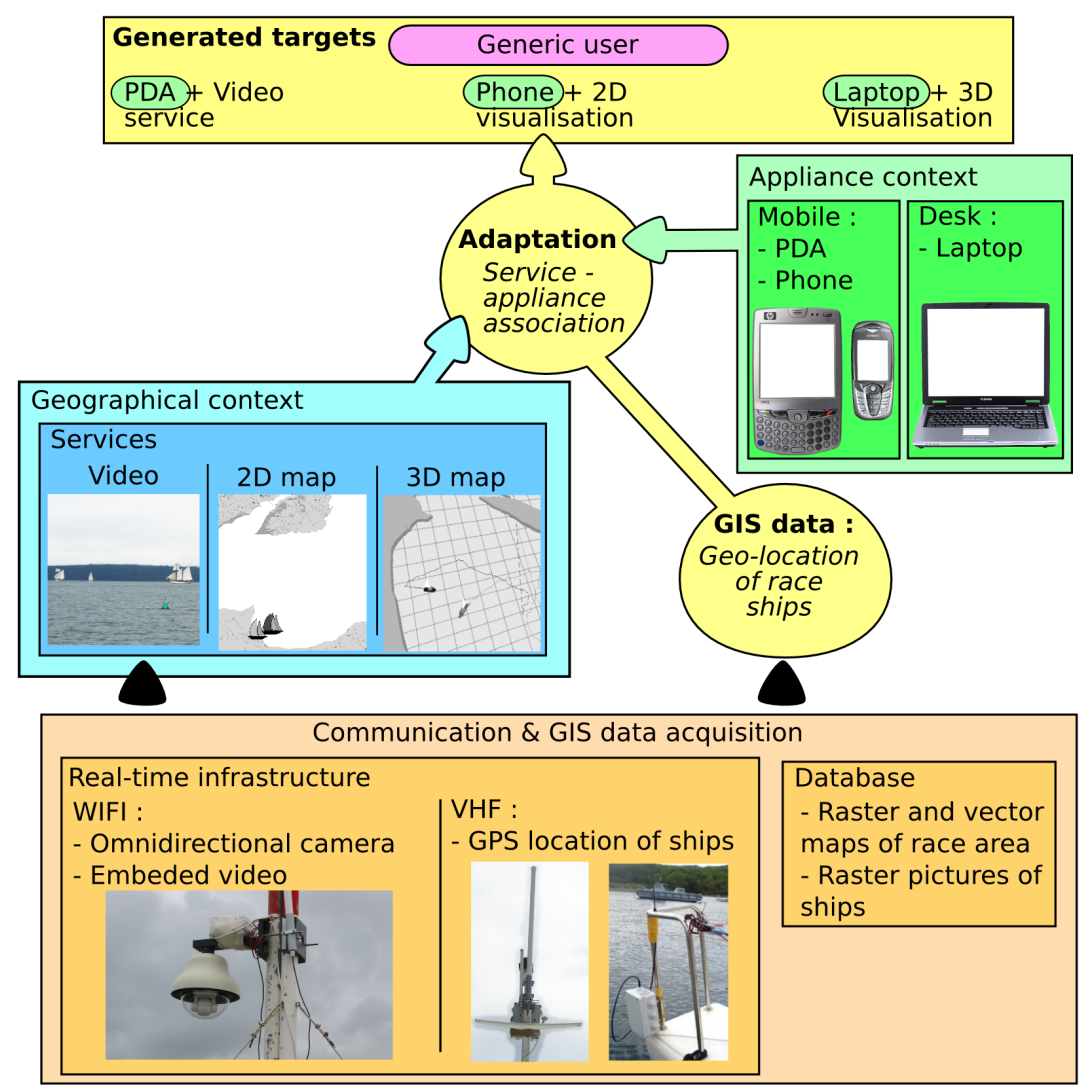

Fig. 5. Experimental implementation of an adaptive GIS model

A general drawback of coastal sailing races is the lack of visibility on the ground, because of the distance to the coast. As real-time positions are crucial, video streams are provided and offer a concrete service of the geographical data. The installed video system presented in Fig. 6- $(e)$ broadcasts a video stream of the race (Fig. 6- $(b))$ to a given WiFi deserved region (Fig. 6- $\left(c_{1}\right)$ ).

\subsection{Services and geographical context}

Different geographical data views, each associated to a particular service, are presented to the user. The " $2 D$ mapping" service delivers ships location information. Different levels of zoom are automatically computed several times per minute to provide detailed views on the race activity. The " $3 D$ mapping" service displays similar data but in a $3 \mathrm{D}$ view of the region of interest. Basic displacement and zooming functions in the scene are available using keyboard combinations. The "Video" service provides a real-time view of the race region. Zooming and camera movements are also allowed. 


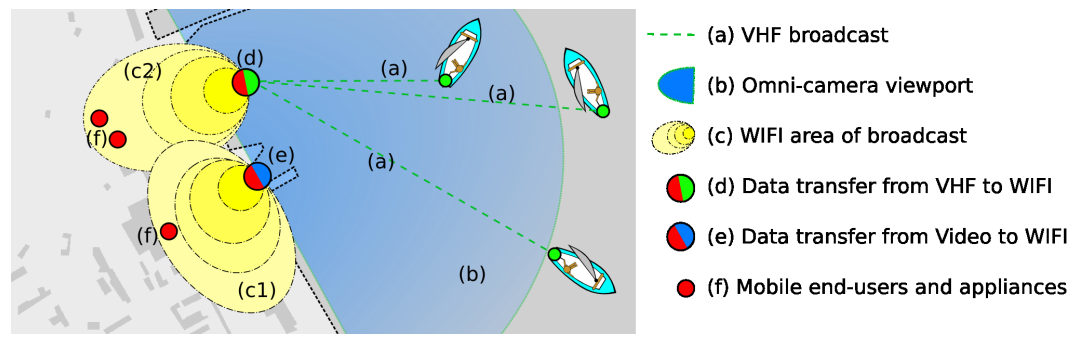

Fig. 6. Real-time communication infrastructure

Each of these three services encompass a geographical context composed of the regions of significance, denoted as $\{U, D, P, S\}_{V i d e o},\{U, D, P, S\}_{2 D},\{U, D$, $P, S\}_{3 D}$ for the user, data, processing and site regions, respectively for "Video", "2D mapping", "3D mapping" services (Fig. 7). As the region of interest is always the racing area, $S_{\text {Video }}=S_{2 D}=S_{3 D}$. The data server for $D_{2 D}$ and $D_{3 D}$ is a computer that collects and stores current and previous ship's locations and spreads the data in a 200 meters wide circular area, that is $D_{3 D}=D_{2 D}$. The data server for $D_{V i d e o}$ and the processing unit for $P_{V i d e o}$ are at the same location (i.e. image acquisition and video signal compression are done together), the resulting video stream is sent wireless over a 200 meters ellipsoid region. $P_{3 D}$ and $P_{2 D}$ are done by the user appliance, at the same location as $U_{2 D}$ and $U_{3 D}$, respectively. The processing region is only local to the appliance. The service view at $U_{V \text { ideo }}$ output videos and allows for the distant manipulation of the camera but no processing is done locally. The users are mobile over space and may intersect or not the data or the processing regions; others regions are fixed (except when $P=U$, that is when processing is done on user's appliance). Two

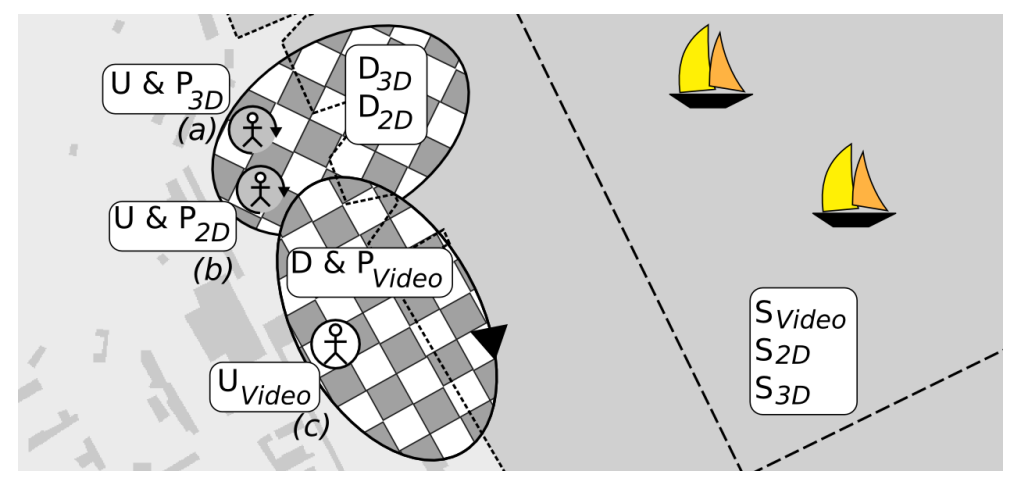

Fig. 7. Geographical context applied to maritime navigation

geographical context changes are identified (Fig.3): 
- For video service, change from $[c, 4]$ to $[c, 1]$

- For 2D and 3D mapping services, change from $[c, 4]$ to $[a, 3]$

These contextual changes are supported by immediate transitions, that is, with no intermediary contextual configurations. However, and in order to ensure a progressive enrichment of the view, intermediate contextual configurations are inserted between initial and final configurations. A given sequence is constructed where two successive configurations differs from one and only one role (Fig. 8). For the video service, these sequences can be represented by $[c, 4] \rightleftarrows[c, 3] \rightleftarrows[c, 1]$ or $[c, 4] \rightleftarrows[c, 2] \rightleftarrows[c, 1]$; whereas for the $2 \mathrm{D}$ and $3 \mathrm{D}$ mapping services, the sequences are $[c, 4] \rightleftarrows[a, 4] \rightleftarrows[a, 3]$ or $[c, 4] \rightleftarrows[c, 3] \rightleftarrows[a, 3]$. Fig. 7 presents the case of the users
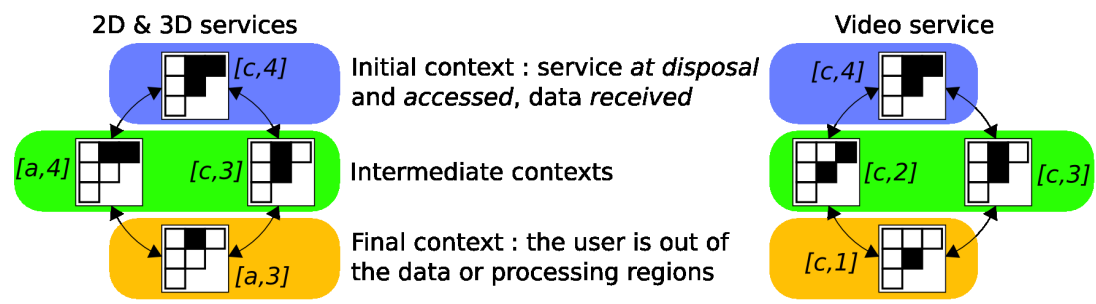

Fig. 8. Transition between geographical contexts

$(a)$ and $(b)$ interacting with two appliances that run the "2D mapping" and "3D mapping" services, respectively. The processing is done at the user's location. $(a)$ and $(b)$ are located in a region where 2D and 3D data are transmitted. When the users leave their region, the intermediate context $[a, 4]$ or $[c, 3]$ occurs simultaneously with the final context $[a, 3]$. A third user $(c)$ is located in a region where the "video" service is available. As $P_{\text {video }}$ and $D_{\text {video }}$ are the same region, when $(c)$ leaves the region, the intermediate contexts $[c, 2]$ or $[c, 3]$ occur simultaneously with the final context $[c, 1]$. The "Video" service view is adapted when contextual configuration switches to $[c, 4]$ or $[c, 1]$ as follows (Fig. 9(a)):

- $[c, 4]$ : the user is located in the region of video streaming. The adaptive GIS is enriched with a new view.

- $[c, 1]$ : as the user is outside the video broadcasting region, the service is not available anymore. On the appliance, no views are provided. The adaptive GIS is waiting for a new service to come up.

The "2D mapping" and "3D mapping" services share the same geographical contexts, adaptation processes and changes at the interface level occur as follows (Fig. 9(b)):

- $[c, 4]$ : the user and the processing services are located in the region of geographical data diffusion, a view showing either a 3D or 2D map is displayed on the device. 
- $[a, 3]$ : the 2D and 3D services run on the user appliance but no data is provided. The service does not update the data anymore. On the appliance side, the view shows the last data received and informs the user that no more data are available for transmission.

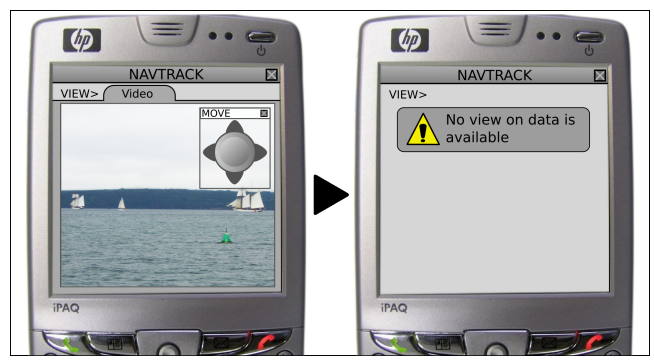

(a) Adaptation to contexts $[c, 4]$ and $[c, 1]$ of the video service

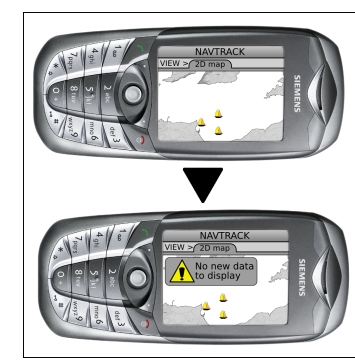

(b) Adaptation to contexts $[c, 4]$ and $[a, 3]$ of the $2 \mathrm{D}$ mapping service

Fig. 9. User-interface adaptation triggered by geographical context changes

The appliance context is also taken into account by the adaptive process. A mapping between the generated view and a particular appliance is applied. The view generated by a "2D mapping" service is displayed on a Java-enabled mobile phone as this service doesn't requires substantial graphical capabilities and as it is adapted to small displays. The " $3 D$ mapping" view is adapted to the desktop PC with sufficient computing power and memory. The "Video" view is displayed on a mobile PDA. Regarding the appliance a user is interacting with, the services are made available or not. For example, if a user with a PDA gets in the " $3 D$ mapping" broadcasting region, no new view can be provided as the associated service is only suitable for users acting on desktop PCs. Fig. 9 shows that the generic user interface is also adapted to the appliance features (e.g. screen size and orientation).

\section{Conclusion and future work}

Although users and appliances are essential components of mobile GISs, they are not always taken into account in the processing and display of geographical data. The research presented in this paper introduces a contextual-based modelling approach that considers users, appliances and geographical data as the core elements of an adaptive GIS. The model proposed identifies and characterises different elements that constitute the geographical context: user, data, process and region of interest. Integration of these elements allows for the identification of different configurations. This influences the way interactions between the user and GIS services should be adapted. This approach is applied to the context of 
maritime navigation and illustrated by a prototype that support the adaptation of the services proposed to the user regarding an evolving geographical context, and the appliance characteristics. Future works concern the integration of the user and appliance contexts and the modelling of evolving configurations using conceptual neighbourhood graphs.

\section{Acknowledgement}

The authors are gratefull to the Science Park Technopole Brest-Iroise for their participation to this work. Special thanks to Yves Vourc'h, Roland Lechallier, Mathieu Goury, Guillaume Mevel and Fabien Nicolas for their help in the implementation of the wireless infrastructure.

\section{References}

1. D.G. Abowd, G.D. Atkeson, J. Hong, S. Long, R. Kooper, and M. Pinkerton. Cyberguide : a mobile context-aware tour guide. In Proceedings of ACM Wireless Networks, pages 421-433. ACM press, 1997.

2. G. Calvary, J. Coutaz, D. Thevenin, Q. Limbourg, L. Bouillon, and J. Vanderdonckt. A unifying reference framework for multi-target user interfaces. Interacting with Computers, 15(3):289-308, 2003.

3. C. Claramunt, S. Fournier, X. Li, and E. Petchev. Real-time geographical information systems for its. In Proceedings of the 5th IEEE International Conference on ITS Telecommunications, pages 237-242, 2005.

4. G. Desvignes, G. Lucas de Couville, E. Peytchev, T. Devogele, S. Fournier, and C. Claramunt. The Share-loc project: a wap-based maritime location system. In B. Huang et al., editor, Proceedings of the 3rd International Conference on Web Information Systems Engineering, pages 88-94. IEEE press, 2002.

5. J. Dubs and R. Kaufmann. Gestion et visualisation de données provenant d'un AIS. Technical report, Naval Academy Research Institute and Université de la Rochelle, 2006.

6. H. Hampe and V. Paelke. Adaptive methods for mobile applications. In Proceedings of the 7th International Conference on Human Computer Interaction with Mobile Devices and Services (MobileHCI 2005), 2005.

7. B. Huang. Developing location-aware navigation guides that uses mobile geographic information systems. Journal of Transportation Research Record, 1879:108-113, 2005.

8. P.A. Longley, M.F. Goodchild, D.J. Maguire, and D.W. Rhind. Geographical Information Systems and Sciences. John Wiley and Sons, 2nd edition, 2005. 517 pages.

9. T. Reichenbacher. Adaptive methods for mobile cartography. In Proceedings of the 21th International Cartographic Conference, pages 1311-1322, 2003.

10. D. Thevenin. Adaptation en Interaction Homme-Machine : le cas de la plasticité. PhD thesis, Université Joseph Fourrier, Grenoble, 2001.

11. A. Zipf. Using styled layer descriptor (SLD) for the dynamic generation of userand context-adaptative mobile maps - a technical framework. In K.J. Li and C. Vangenot, editors, Proceedings of the 5th International Workshop on Web and Wireless GIS (W2GIS 2005), pages 183-193. Springer-Verlag, 2005. 